\title{
Recherche d'une physique au-delà du modèle standard et étude de la violation de symétrie CP avec l'expérience LHCb au CERN
}

Frédéric Machefert (frederic.machefert@in2p3.fr)

Laboratoire de l'accélérateur linéaire (LAL, UMR8607), Centre scientifique d’0rsay, Bât. 200, BP 34, 91898 Orsay Cedex

LHCb est l'une des expériences situées sur l'anneau de collision de protons du CERN, le LHC. Son objectif est d'explorer les légères différences qui existent entre matière et antimatière, et de mesurer des taux de désintégrations rares de particules.

Les données accumulées ces dernières années fournissent de nombreux résultats,

en particulier sur la mesure de l'angle $\gamma$ du triangle d'unitarité CKM, sur les propriétés des mésons B et D, et sur les désintégrations des mésons B. Elles donnent ainsi un premier éclairage sur ce que pourrait être (ou non) une physique au-delà du modèle standard.
La physique des particules est l'étude des constituants élémentaires de la matière et de leurs interactions. L'un des moyens les plus efficaces pour approfondir les connaissances dans ce domaine est la réalisation d'expériences de collisions de particules. Le Large Hadron Collider (LHC) du CERN, le laboratoire européen pour la physique des particules, est un anneau de $27 \mathrm{~km}$ de circonférence dans lequel circulent en sens opposés deux faisceaux de protons. L'énergie et la fréquence des collisions aux quatre points de croisement des faisceaux atteignent des niveaux sans précédent, qui permettent de fournir aux expériences des données d'une grande richesse.

Deux détecteurs généralistes, ATLAS et CMS, ont essentiellement pour but de produire directement et d'étudier le boson de Higgs et de possibles nouvelles particules. Les deux autres expériences sont ALICE, dédiée à la recherche d'un nouvel état dense et chaud de la matière, le plasma de quarks et de gluons, et $\mathrm{LHCb}$ qui étudie la physique des différents types (ou «saveurs") de quarks et les interactions entre quarks de différentes familles. Ce domaine est relativement vaste et, à l'instar des deux expériences généralistes déjà citées, $\mathrm{LHCb}$ (dont le détecteur est décrit dans l'encadré 1, p. 20) a également pour but de mettre en évidence, de manière indirecte, une nouvelle physique au-delà du "modèle standard ", qui réunit l'essentiel de nos connaissances en physique des particules.

\section{Le modèle standard}

Le modèle standard repose sur la relativité restreinte et la mécanique quantique, puisqu'il décrit des objets rapides et petits. Les phénomènes de création et d'annihilation de particules ont obligé les théoriciens à développer les outils mathématiques de la théorie des champs, afin de décrire des populations de particules dont le nombre n'est pas fixe. Enfin, un élément essentiel en physique des particules est l'intégration de la notion de symétrie de jauge locale, qui permet de rendre invariantes les équations par rapport à des changements arbitraires de variables (temps, coordonnées spatiales, vitesses...). Ces symétries sont l'expression de la conservation de certaines grandeurs, comme les charges. Les "équations " qui découlent de ces fondements sont ensuite appliquées au contenu du modèle standard : les particules.

Le modèle standard décrit la matière avec douze fermions de spin $1 / 2$ : six leptons et six quarks (voir tableau 1). La charge électrique des quarks « $\mathrm{u}$ », « $\mathrm{c}$ » et « $\mathrm{t}$ » (ligne supérieure du tableau) est $+2 / 3$, alors que celle des quarks « $\mathrm{d}$ ", « s » et « b " (ligne inférieure pour les quarks) est $-1 / 3$. Les fermions sont groupés par familles (ou colonnes sur le tableau 1), contenant chacune deux leptons et deux quarks. La principale différence entre les familles tient dans les masses des particules qui les composent. Les interactions entre ces fermions sont 


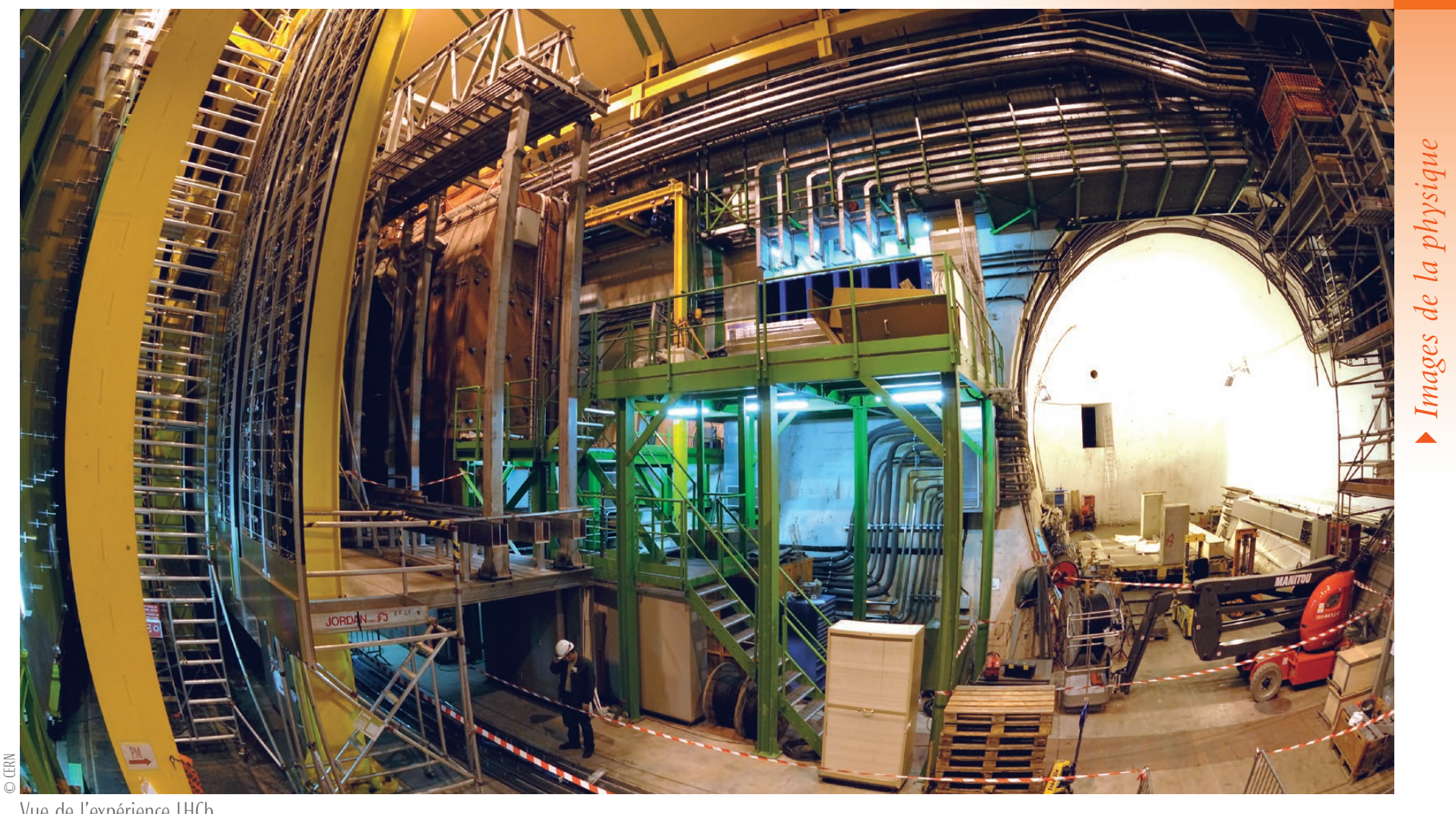

Vue de l'expérience LHCb.

vues comme des échanges de particules, des bosons de spin 1, vecteurs des forces ou particules d'interactions et appelés photon pour l'électromagnétisme, bosons $\mathrm{Z}$ et $\mathrm{W}$ pour l'interaction faible, et gluons pour la force forte ${ }^{(a)}$. Par exemple, la répulsion de deux électrons dont la charge électrique est négative est interprétée comme l'échange de photons entre ces deux particules.

Le modèle standard étend la notion de charge, commune en électromagnétisme, à l'ensemble des forces. La charge électrique caractérise les particules sensibles à l'interaction électromagnétique. De manière identique, d'autres charges sont liées aux deux autres forces. Mais pour ces dernières, les bosons vecteurs (le Z, les W et les gluons) portent également des charges et peuvent donc interagir avec d'autres bosons du même type, un peu comme si on imaginait un photon portant une charge électrique. À partir de cet alphabet relativement simple, il est possible de fabriquer la matière qui nous entoure en associant les fermions, le ciment étant constitué par les bosons. Une dernière particule complète ce tableau : il s'agit du boson de Higgs (particule de spin 0). Il est intégré au modèle standard depuis longtemps, mais n'a été observé directement au LHC qu'en 2012, sa mise en évidence apportant le prix Nobel de physique 2013 à Englert et Higgs pour leur prédiction théorique. Cette particule et le champ qui

Récapitulatif des particules du modèle standard.

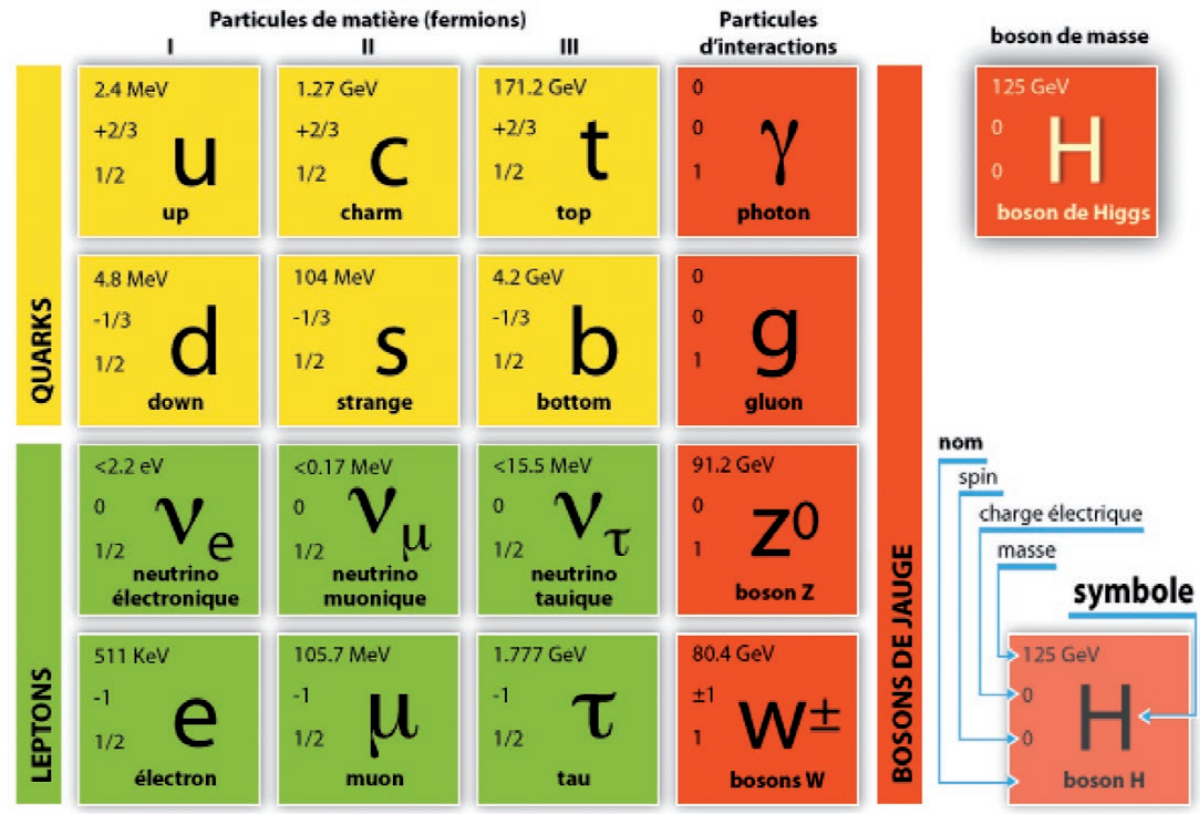

Tableau 1. Les quarks sont en jaune et les leptons en vert. Ils constituent la matière et sont groupés par familles. Les trois familles connues correspondent aux colonnes notées I, II et III. Les autres particules (en rouge) sont les bosons vecteurs des interactions, et le boson de Higgs.

lui est associé permettent de fournir de manière élégante leurs masses aux fermions et aux bosons du modèle, en conservant son pouvoir prédictif et sa cohérence.

Enfin, pour clore cette description du modèle standard, il faut garder à l'esprit que chacun des fermions mentionnés plus haut apparaît sous deux aspects différents, comme particule de matière et comme particule d'antimatière (pour lesquelles les signes des charges sont opposés), doublant du coup le nombre de fermions.

Conventionnellement, et cette notation sera suivie ici, la particule d'antimatière est indiquée avec une barre surmontant le symbole attribué à la particule de matière. 
$>>$

Cette image cohérente masque un certain nombre de difficultés occultées ou imparfaitement résolues par le modèle actuel. L'une d'elles est la différence observée entre les quantités de matière et d'antimatière dans l'univers. On suppose qu'à l'origine l'univers était symétrique. Un processus a dû rompre l'équilibre entre les deux formes, notre univers étant essentiellement vide, avec une faible quantité de matière et sans antimatière. Le physicien russe Sakharov a été le premier à énoncer, en 1967, les trois conditions nécessaires à ce déséquilibre, l'une d'elles étant la violation de la symétrie CP.

\section{La violation de la symétrie CP et les désintégrations rares}

En mécanique quantique, l'opération « $\mathrm{CP}$ » consiste à appliquer les opérateurs $\mathrm{C}$ et $\mathrm{P}$, qui renversent respectivement les charges et l'orientation de l'espace (un peu comme le ferait un miroir). Appliquer ces opérateurs $\mathrm{C}$ et $\mathrm{P}$ sur la transition d'un état initial vers un état final, $\mathrm{CP}($ Initial $\rightarrow$ Final $)$, consiste à regarder cette transition pour les états symétriques matière-antimatière associés, $\overline{\text { Initial }} \rightarrow \overline{\text { Final. Sans violation de }}$ la symétrie CP, les intensités de ces deux transitions sont identiques. Une violation suppose un traitement asymétrique de la matière et de l'antimatière par la Nature.

Les violations des symétries $\mathrm{C}$ et $\mathrm{P}$, appliquées indépendamment, sont connues depuis longtemps. La violation de la symétrie $\mathrm{CP}$ a été plus difficile à observer, en raison de la faible intensité du phénomène. Le premier secteur où l'effet a été vu est celui du quark de saveur étrange «s " (de l'anglais "strange") de la deuxième famille (voir le tableau 1), lors de réactions impliquant les mésons (qui sont des particules fabriquées à partir d'une paire quark-antiquark) appelés K, dont l'un des deux constituants a la saveur «s ». Au début des années 2000, les expériences Babar et Belle, respectivement aux États-Unis et au Japon, ont étendu l'exploration au secteur du quark « b » (de l'anglais “bottom"), appartenant à la troisième famille, plus difficile à produire mais où la théorie prévoit une intensité plus forte du phénomène.
L'observation de cette asymétrie a valu le prix Nobel à des expérimentateurs ainsi qu'aux théoriciens qui ont su l'intégrer au modèle standard (encadré 2). Pourtant, le modèle standard est clairement mis en défaut dans la nature, puisque l'amplitude de la violation de CP nécessaire pour rendre compte de l'asymétrie dans l'univers, est de plusieurs ordres de grandeur supérieure à ce que nous observons actuellement et à ce qui est calculé. Parallèlement, ces failles indiquent que l'étude poussée de la violation de CP devrait fournir plus facilement des indications sur une physique nouvelle, au-delà du modèle standard, et donnent une grande importance à ce domaine de recherche.

La notion de violation de la symétrie CP est étroitement liée à la saveur des quarks, ce dont rend compte le modèle standard qui intègre l'effet (voir encadré 2). L'étude des mésons B (dont l'un des constituants est de saveur " $\mathrm{b} \|)$ est le domaine le plus favorable à l'étude de la violation de CP. Les conditions du faisceau, notamment l'énergie du LHC et la zone angulaire

\section{Le détecteur $\mathrm{LHCb}$}

LHCb est une collaboration internationale regroupant plus de 700 personnes provenant de 52 universités à travers le monde, qui ont conçu le détecteur et, depuis 2010, exploitent ses données.

Le détecteur LHCb est situé sur l'anneau de collision du LHC et bénéficie de la grande probabilité de production de paires de quarks « $b$ » " $\bar{b}$ » aux énergies du LHC, lors des collisions proton-proton. La configuration du détecteur (fig. E1), dont I'ouverture angulaire rend possible l'observation des particules produites près de l'axe des faisceaux, permet de suivre les trajectoires des particules issues de la désintégration des mésons B.

Après la collision, les particules produites par interaction protonproton, ou plus exactement par interaction de quarks ou de gluons, constituants élémentaires des protons, traversent le détecteur de "vertex ». Ce détecteur, constitué de plans de silicium, mesure avec une très grande précision les trajectoires des particules chargées. Il permet notamment de déterminer très précisément le point de désintégration des hadrons contenant un quark « b». Ces particules composées ont une durée de vie relativement longue, ce qui permet de les identifier par les distances, de l'ordre du centimètre, séparant le point d'interaction des protons, lieu de production de ces hadrons, de ceux de leurs désintégrations. Ensuite, les particules traversent le reste du trajectographe de l'expérience, qui mêle des plans de mesure de la trajectoire avec un champ magnétique intense, et fournit la mesure de l'impulsion des particules chargées. L'identification des particules est faite par deux imageurs à effet Cherenkov, sensibles à la masse des particules, les calorimètres (qui mesurent l'énergie des photons, des électrons et des hadrons) et les détecteurs à muons.

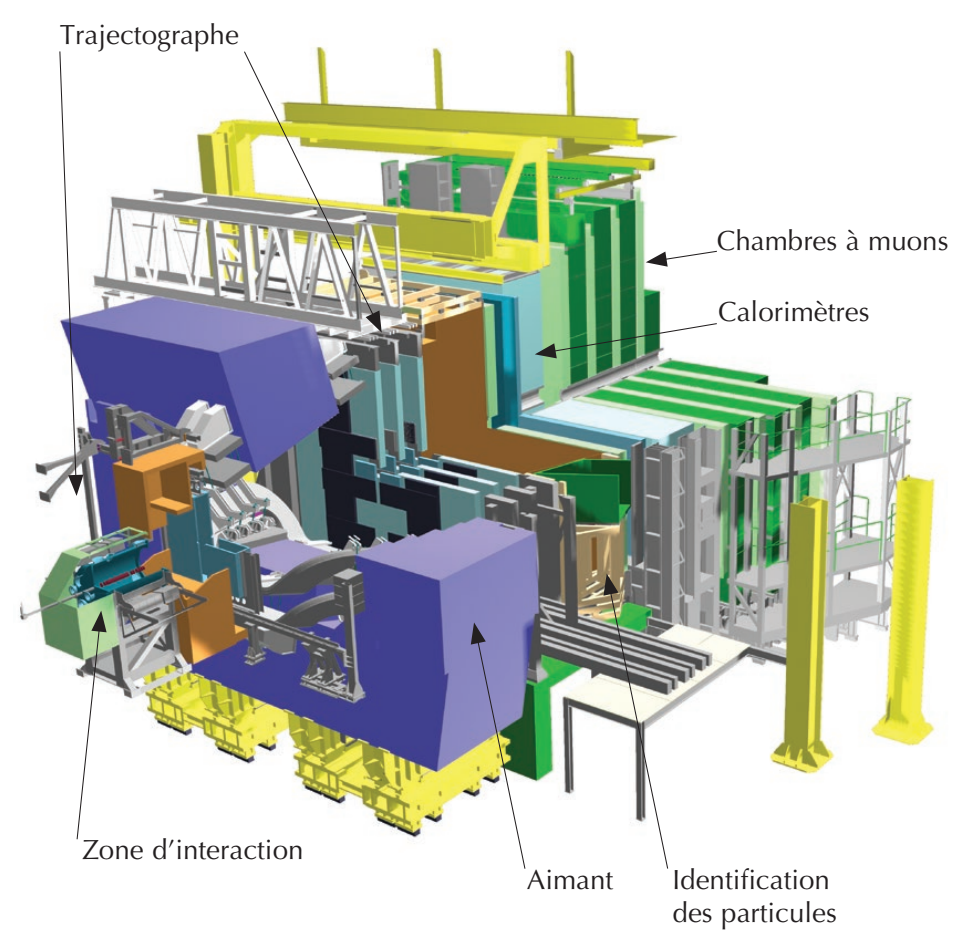

E1. Schéma du détecteur LHCb, montrant le point d'interaction proton-proton et les principaux éléments de détection des particules produites. 
couverte par le détecteur $\mathrm{LHCb}$, permettent la production et l'observation d'un grand nombre d'événements contenant des quarks « b " à partir d'une collision proton-proton. Au point d'interaction de l'expérience, environ $10^{12}$ paires $《 \mathrm{~b} »\langle\overline{\mathrm{b}}$ » sont produites chaque année. Seule une fraction de ces événements est intéressante et sera stockée sur bande pour l'analyse. Mais cela constitue le plus large échantillon de données de ce type jamais enregistré. Il s'agit ici du premier grand axe de recherche de l'expérience $\mathrm{LHCb}$.

La statistique accumulée chaque année par LHCb permet d'étendre naturellement le domaine de recherche de l'expérience à un second axe, le secteur des désintégrations rares. Les prédictions du modèle standard sont, dans un très grand nombre de situations, très précises. Notamment, certaines transitions sont interdites ou très peu probables. Il s'agit souvent de modes de désintégration dont la forme simple (dite à " l'arbre ", fig. E2a) fait intervenir un terme très petit du modèle standard ou bien viole une règle de conservation. Les diagrammes (voir encadré 3) alors autorisés sont plus complexes, avec des boucles de particules (certains de ces diagrammes sont dits " en boîte ", le chemin des particules sur la figure formant un carré comme sur la figure E2c, ou "pingouins » en raison de leur forme également, voir la figure 1, et si vous avez beaucoup d'imagination !). Ces derniers conduisent en général à une faible fréquence des événements recherchés. Mais, par les boucles qu'ils contiennent, ces diagrammes ont un intérêt particulier, notamment dans le cadre de nouvelles physiques au-delà du modèle standard.

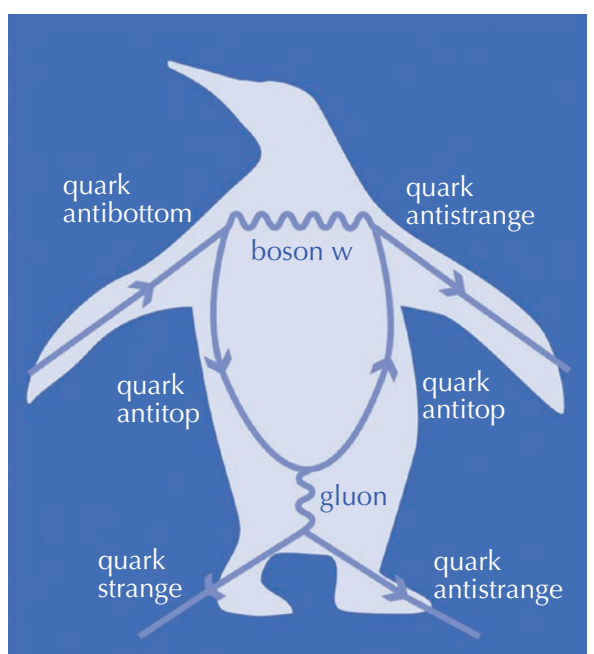

\section{Le modèle standard et la violation de la symétrie CP}

La violation de la symétrie $\mathrm{CP}$ est incluse indirectement dans le modèle standard. Elle tient son origine de la force faible et des courants chargés associés, c'est-à-dire des interactions dont le médiateur est le boson doté $\mathrm{d}^{\prime}$ une charge électrique, $\mathrm{W}^{+} \mathrm{W}^{-}$. Le modèle standard stipule que les états propres des quarks, vus par l'interaction faible, ne sont pas les états propres de masse (les états physiques). Ainsi, lorsqu'un boson W interagit avec un quark, il ne voit pas un état de saveur du type de ceux indiqués dans le tableau 1, mais une combinaison linéaire, une superposition de deux états.

Les transitions autorisent les changements de saveur lors de l'interaction, et la probabilité des couplages entre un boson $W$ et deux quarks de saveurs données est fournie par la matrice de rotation entre les états propres de masse et les états vus par l'interaction faible. Cette matrice, appelée communément « matrice CKM » d'après les noms des trois théoriciens à l'origine du modèle, N. Cabibbo, M. Kobayashi et T. Maskawa, est de dimension $3 \times 3$ dans le modèle standard (parce que ce modèle intègre trois familles) et permet de coupler les quarks chargés positivement avec les quarks de charge négative. Ainsi, par exemple, un « $d$ » se couple avec un " $u$ » avec une amplitude $V_{\text {ud }}$.

$$
\hat{\mathrm{V}}_{\mathrm{CKM}}=\left(\begin{array}{lll}
\mathrm{V}_{\mathrm{ud}} & \mathrm{V}_{\mathrm{us}} & \mathrm{V}_{\mathrm{ub}} \\
\mathrm{V}_{\mathrm{cd}} & \mathrm{V}_{\mathrm{Cs}} & \mathrm{V}_{\mathrm{cb}} \\
\mathrm{V}_{\mathrm{td}} & \mathrm{V}_{\mathrm{ts}} & \mathrm{V}_{\mathrm{tb}}
\end{array}\right)
$$

La matrice CKM rend compte de la force des couplages entre les quarks via l'interaction faible et montre une hiérarchie intéressante entre ces couplages. Les termes diagonaux sont très proches de l'unité, ce sont les couplages les plus favorisés. Ils sont de plus en plus petits lorsqu'on s'éloigne de la diagonale.

Mais le formalisme de la matrice CKM va beaucoup plus loin et a d'importantes implications en physique des particules. Les propriétés de cette matrice sont celles d'une matrice de rotation et elle doit vérifier les relations mathématiques correspondantes. Ainsi, le produit avec son adjointe doit être l'identité. Cette contrainte réduit le nombre de degrés de liberté de la matrice, qui dépend de sa dimension et donc du nombre $\mathrm{N}$ de familles et vaut $(\mathrm{N}-1)^{2}$, à savoir $\mathrm{N}(\mathrm{N}-1) / 2$ angles de rotation et $(\mathrm{N}-1)(\mathrm{N}-2) / 2$ phases complexes. Pour deux familles, il n'existe qu'un angle de rotation, appelé angle de Cabibbo. À l'origine, la matrice ne contenait que ce paramètre. Mais les expériences ont pu déterminer l'existence $d^{\prime}$ 'une troisième famille intégrée au modèle standard, et la matrice s'est enrichie de deux nouveaux angles de rotation et d'une phase complexe.

La phase complexe $\eta$ est à l'origine de la violation de la symétrie CP dans le modèle standard. Supposons une désintégration faisant appel à l'interaction faible et donc pour laquelle I'amplitude est liée à un terme de la matrice CKM. Ce terme et I'amplitude correspondante dépendent potentiellement de la phase $\eta$. Mais, à l'instar d'une expérience de mécanique quantique habituelle, lorsqu'une seule amplitude intervient, les phases complexes disparaissent lorsque l'intensité du phénomène est calculée. En d'autres termes, l'observable physique ne dépend pas de la phase. Cette situation est plus complexe lorsque (et c'est le cas dans les transitions qui nous intéressent ici) plusieurs amplitudes, correspondant à différents chemins pour aller de l'état initial à l'état final, contribuent à la désintégration. Alors, l'intensité dépend de la phase complexe de la matrice CKM de par les interférences entre amplitudes. Cette phase change de signe lorsqu'on considère les antiparticules. Le modèle standard, par la phase complexe de la matrice CKM et les interférences, intègre ainsi la violation de la symétrie CP.

$C^{\prime}$ est un succès pour ce modèle. Pourtant, il s'avère que le phénomène calculé par les équations du modèle standard, s'il correspond à ce qui est mesuré par nos expériences, est plusieurs ordres de grandeur en-dessous de ce dont nous aurions besoin pour expliquer l'état actuel de notre univers. La réponse actuelle est au mieux une ébauche et notre compréhension n'est que partielle. C'est pourquoi ce domaine de recherche est très actif et est considéré comme l'une des pistes les plus sérieuses à suivre pour découvrir une nouvelle physique au-delà du modèle standard.

1. Représentation d'un diagramme de Feynman, dit « pingouin ». Les particules entrent par la gauche, et celles qui sortent par la droite ont été produites par la réaction. La boucle intermédiaire contient des particules « virtuelles », susceptibles d'avoir des masses élevées et d'être encore inconnues. 


\section{Les diagrammes de Feynman} encadré 3

Le physicien Richard Feynman a introduit, à la fin des années 1940, des diagrammes qui permettent de représenter la propagation des particules élémentaires, leurs interactions et leurs désintégrations. Ils donnent une représentation compacte et plus facile à interpréter des processus intervenant en physique des particules. La propagation des particules est figurée par des lignes (droites pour les fermions, ondulées pour les bosons), qui traduisent schématiquement l'amplitude de probabilité qu'a une particule d'aller d'un endroit à un autre. Les points où les lignes se rencontrent (communément appelés " vertex ») correspondent à deux ou plusieurs particules qui se trouvent être au même endroit au même moment, et matérialisent leur interaction. Habituellement, sur les diagrammes, le temps va de gauche à droite et la dimension spatiale est verticale. Les diagrammes de Feynman peuvent avoir une forme simple, dite " en arbre » (fig. E2a), ou une forme plus complexe, avec une «boucle» (fig. E2b) ou en «boîte» (fig. E2c).

Il est possible d'appliquer des règles de calcul, les règles de Feynman, à chaque partie d'un diagramme (lignes internes et externes, vertex, etc.) afin de déterminer l'amplitude correspondante du phénomène. $\mathrm{a}$

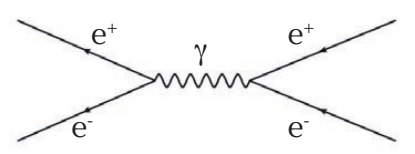

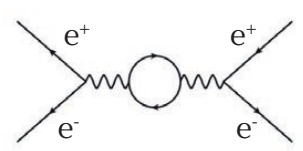

$\mathrm{b}$

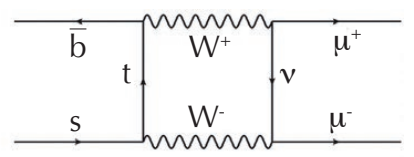

C
E2. Exemples de diagrammes de Feynman : une paire électron-positron s'annihile en donnant un photon qui produit à nouveau une paire électron-positron (a) ; une autre contribution à ce processus physique avec les mêmes états initiaux et finals, mais incluant une boucle correspondant à l'apparition et l'annihilation de particules virtuelles de très courte durée de vie (b) ; et, enfin, le diagramme de production d'une paire de muons par la désintégration d'un méson $B_{s}$ dans le cadre du modèle standard (c).

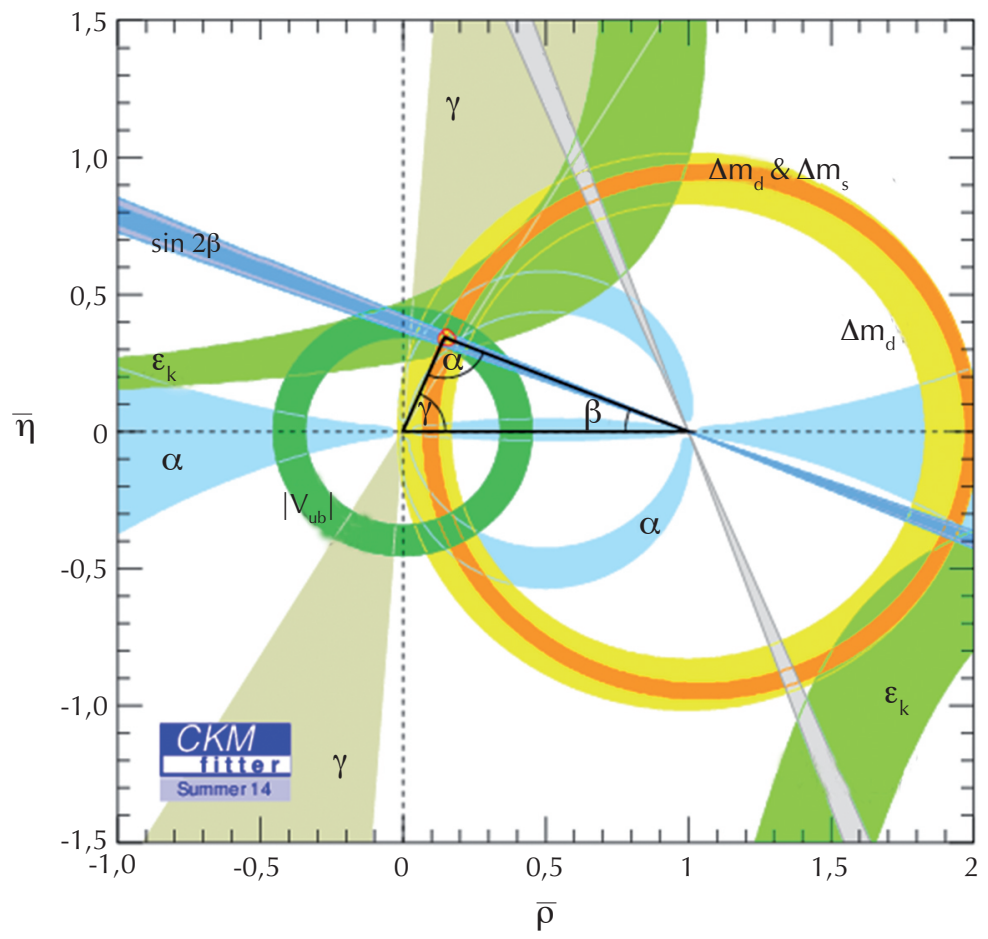

2. Le triangle d'unitarité (en noir), obtenu en combinant les mesures actuelles sur la violation de CP, qui correspondent aux différents domaines colorés : leur intersection, non vide, définit les régions permises pour le sommet du triangle. En ordonnée, la phase $\eta$ à l'origine de la violation de symétrie (P dans le modèle standard (voir l'encadré 2). Des trois angles du triangle, $\gamma$ est le moins bien déterminé. $\gg>$

Le principe d'incertitude d'Heisenberg, bien connu en mécanique quantique, s'applique en physique des particules. Il stipule que l'énergie d'une particule dont l'existence est brève (on dit alors que la particule est " virtuelle ») n'est pas bien définie et peut donc potentiellement être grande. Les particules circulant dans les boucles (fig. E2b) des diagrammes complexes sont virtuelles (elles n'apparaissent pas dans l'état final et disparaissent juste après leur apparition), leur énergie n'est donc plus aussi fortement contrainte que pour les diagrammes simples, dits à "l'arbre ". On peut supposer que des particules liées à une physique au-delà du modèle standard et de forte masse peuvent circuler dans ces boucles. Sans qu'elles puissent apparaitre dans l'état final (elles doivent être virtuelles), leur effet est potentiellement visible dans de très nombreuses observables (les distributions angulaires lors de réactions, le nombre d'événements d'un certain type, etc.). Au-delà de la compréhension de la violation de $\mathrm{CP}, \mathrm{LHCb}$ effectue de nombreuses mesures liées à ces effets, la collaboration ayant à ce jour publié plus de 200 articles. Certaines sont décrites ici.

\section{Le triangle d'unitarité}

Comme évoqué plus haut, un mécanisme inclus dans le modèle standard et décrit dans l'encadré 2, permet de rendre compte des transitions entre familles et de la violation de CP. Ce mécanisme et les paramètres qu'il utilise peuvent être combinés et représentés dans le plan complexe sous la forme d'un triangle appelé triangle d'unitarité, qui aide à visualiser toute incompatibilité entre les mesures, toujours dans le cadre du modèle standard (fig. 2).

Ainsi, le triangle doit être fermé, les rapports entres les côtés et les angles doivent être en accord, etc. Une incohérence entre deux grandeurs est potentiellement l'indication d'une lacune du modèle standard et a son origine dans des phénomènes nouveaux. Jusqu'à présent, l'accord est très bon entre les mesures. Mais on peut supposer que cela est lié à la méconnaissance de certaines quantités, autrement dit aux erreurs de mesure actuelles. L'un des objectifs est donc d'améliorer la précision sur ces grandeurs.

Parmi les angles du triangle, $\gamma$ est le moins bien connu (fig. 2). Les expériences actuelles déterminent essentiellement cet angle par l'étude du canal de désintégration $\mathrm{B}^{ \pm} \rightarrow \mathrm{DK}^{ \pm}$, 
où un méson $\mathrm{B}$ chargé (constitué d'une paire quark-antiquark « $b$ » et « $u$ ») produit deux mésons, D (contenant un quark " c ») et $\mathrm{K}$ (avec un quark de type "s "). Deux diagrammes à l'arbre contribuent principalement à cette désintégration et font apparaître, sous la forme d'une phase relative, l'angle $\gamma$.

Après deux années complètes de fonctionnement, $\mathrm{LHCb}$ a accumulé suffisamment de données pour extraire cet angle avec une précision de l'ordre de celle des expériences précédentes, et conduisant à une incertitude sur la moyenne mondiale de $10^{\circ}$ pour $\gamma$. L'accumulation des données avec $\mathrm{LHCb}$ doit permettre dans les années à venir de réduire à quelques degrés l'imprécision sur cette mesure.

D'autres méthodes, basées sur des diagrammes avec des boucles, devraient également fournir des résultats dans les années à venir. Ces diagrammes en boucles, on l'a vu, sont plus sensibles à une nouvelle physique. Une différence entre les mesures de l'angle $\gamma$ par les diagrammes « à l'arbre » et par les diagrammes en boucle serait également un indice important pour l'existence d'une physique au-delà du modèle standard.

\section{Les oscillations des mésons B et D}

Les mésons neutres, dits $\mathrm{B}_{\mathrm{d}}$ et $\mathrm{B}_{\mathrm{s}}$ (constitués d'un antiquark « $\bar{b}$ » et respectivement d'un quark « d » et "s »), subissent un phénomène remarquable où, au cours de sa propagation, la particule peut se transformer en sa propre antiparticule et vice versa. On observe alors une " oscillation » d'une particule vers son antipartenaire, jusqu'à sa désintégration. Cet effet est possible via des diagrammes en boîte (fig. E2c), très sensibles à une physique nouvelle et dont les paramètres peuvent être reliés au mécanisme de violation de $\mathrm{CP}$.

Ces oscillations ont été observées dans un premier temps par les expériences du LEP, l'accélérateur qui a précédé le LHC au CERN, puis par l'expérience CDF au Tevatron, aux États-Unis, pour le $B_{d}$ et le $\mathrm{B}_{\mathrm{s}}$, respectivement. $\mathrm{LHCb}$ a mesuré avec une exceptionnelle précision ces oscillations. La figure 3 montre, en fonction de leur temps de vol avant désintégration, le nombre de mésons $\mathrm{B}$ qui se désintègrent dans le même état ou dans l'état opposé à celui de leur production. Certains paramètres du modèle standard associés à ces oscillations sont déterminés à partir des points de mesure et fournissent les courbes théoriques superposées aux points sur la figure 3 , contraignant par ailleurs fortement toute déviation par rapport au mécanisme standard.

Malgré son nom, l'expérience LHCb étudie également le quark "c » (pour " charme ") qui, à l'image du «b », est produit en grand nombre au point de collision. Cette abondance a permis d'observer pour la première fois avec certitude l'oscillation du méson D contenant ce quark « $\mathrm{c}$ ».

\section{La désintégration $\mathrm{B}_{\mathrm{S}} \rightarrow \mu^{+} \mu^{-}$}

Le méson $\mathrm{B}_{\mathrm{s}}$ ne peut que difficilement se désintégrer en une paire de muons dans le cadre du modèle standard (de l'ordre de trois désintégrations de ce type pour un milliard de désintégrations du $\mathrm{B}_{\mathrm{s}}$ ) ; mais une variété de modèles (notamment supersymétriques [4]), décrivant une physique nouvelle, prédisent une augmentation souvent sensible, voire drastique (pouvant atteindre plusieurs ordres de grandeur), du taux de ces désintégrations. Ce canal, par ses capacités à tester nos connaissances et les extensions au modèle standard, a été pendant longtemps l'un des phénomènes les plus recherchés en physique des particules, donnant à ce champ d'investigation une importance considérable.

Dès 2012 et avec une statistique partielle, $\mathrm{LHCb}$ avait pu détecter des désintégrations s'effectuant par ce canal (fig. 5, p. 24). La mesure est maintenant confirmée par $\mathrm{LHCb}$ (en utilisant un échantillon de données plus important) et CMS, l'une des trois autres expériences du LHC. La figure 4 montre la masse invariante reconstruite à partir de la paire de muons et pour les événements sélectionnés par l'analyse des données. Une légère accumulation est visible autour de $5,4 \mathrm{GeV} / \mathrm{c}^{2}$, valeur correspondant à la masse du $\mathrm{B}_{\mathrm{s}}$. La mesure est en parfait accord avec le modèle standard, mais elle permet par ailleurs de contraindre, comme rarement une unique mesure, de nombreux modèles de nouvelle physique.

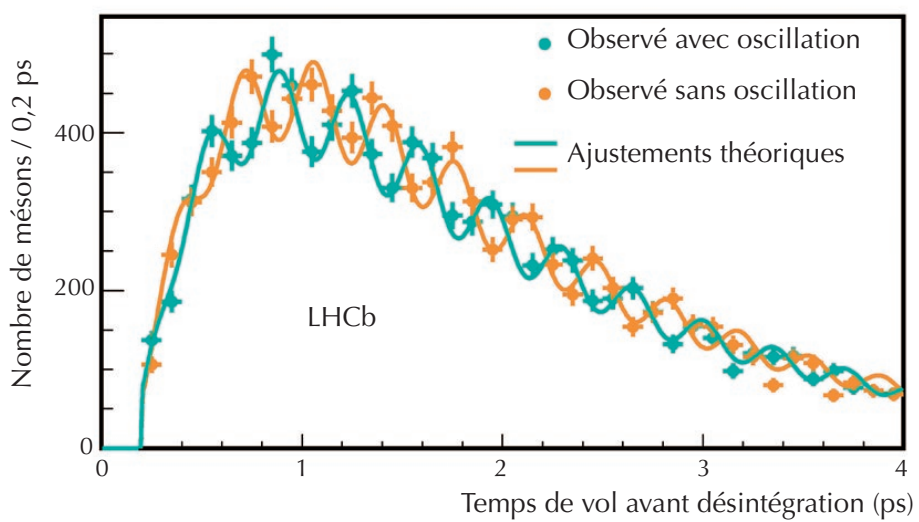

3. Nombre de mésons B sélectionnés au cours de l'analyse de données, en fonction de la durée de vol des particules avant désintégration. En orange, sont indiqués les mésons mesurés lors de leur désintégration dans le même état que celui dans lequel ils ont été produits ; en vert, ceux qui ont changé de saveur au moment de la désintégration.

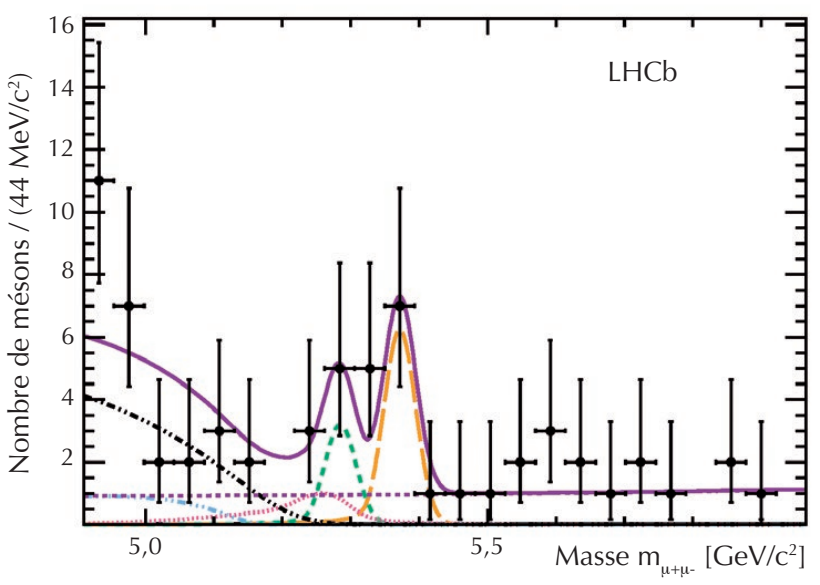

4. Masse reconstruite (en abscisse) à partir des deux muons observés et pour les candidats sélectionnés par l'analyse des événements du type $B_{s} \rightarrow \mu^{+} \mu^{-}$. Les points avec leurs barres d'erreur sont les données et indiquent une accumulation autour de $5,4 \mathrm{GeV} / \mathrm{c}^{2}$, correspondant à la masse du $B_{s}$. Les courbes de couleur en tirets et pointillés représentent les contributions attendues pour les bruits de fond et, en orange, le signal recherché. La courbe violette est la somme attendue de toutes ces contributions. 


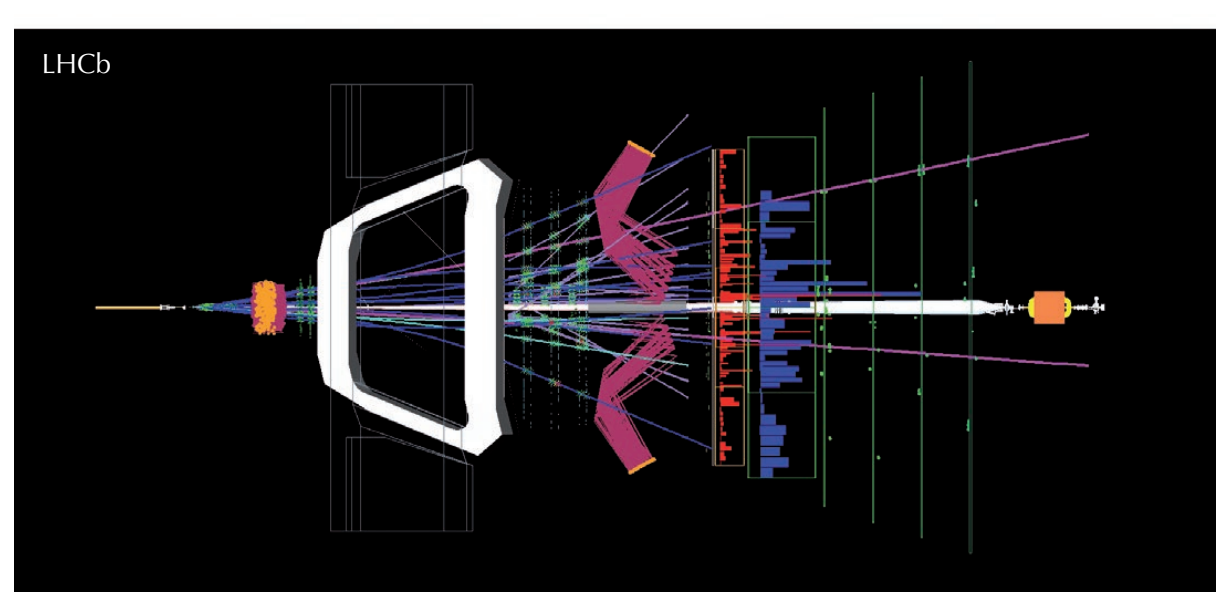

5. L'image montre un événement compatible avec la désintégration d'un méson $B_{\mathrm{s}}$ en une paire de muons $\mu^{+}$et $\mu^{-}$. La paire de muons s'échappe par l'arrière du détecteur (à droite de la figure). La position du point de collision proton-proton est reconstruite par la convergence des traces mesurées dans le détecteur. Les deux muons sont indiqués par des trajectoires violettes. Le méson $B_{\varsigma}$ n’est pas vu dans le détecteur, mais sa direction et sa longueur de vol (de l'ordre du centimètre) par rapport au point d'interaction sont déterminées grâce aux muons.

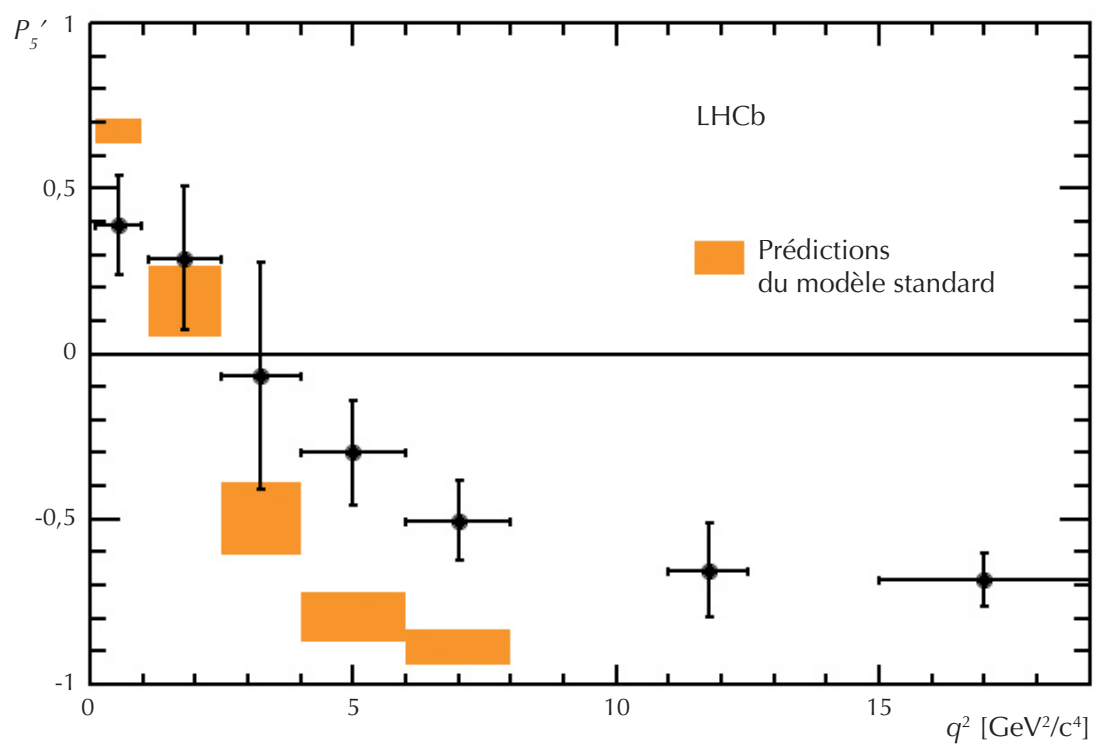

6. Le paramètre $P_{5}$ ' lié aux distributions angulaires observées par LHCb lors de désintégrations du type $B \rightarrow K^{*} \mu^{+} \mu^{-}$(points avec les barres d'erreur) est comparé avec les prédictions du modèle standard (en orange, la hauteur des rectangles indique la précision des calculs). Le paramètre $q^{2}$ est le carré de la masse invariante de la paire de muons.

(a) La force gravitationnelle a une intensité trop faible pour avoir un effet mesurable dans les expériences actuelles en physique des particules sur accélérateur et rentre mal dans le cadre théorique. Elle n'est pas prise en compte dans le modèle standard.

\section{En savoir plus}

1- Le site public de LHCb

http://Ihcb-public.web.cern.ch/Ihcb-public/

2 - Le site français dédié au LHC et aux expériences www.lhc-france.fr

3• L. di Ciaccio et G. Hamel de Monchenault, "Découverte du Boson de Higgs au LHC?», Reflets de la physique n³1 (2012) 17.

\section{Le canal $B \rightarrow K^{*} \mu^{+} \mu^{-}$}

La plupart des résultats obtenus par $\mathrm{LHCb}$ ont à la fois amélioré la précision sur de nombreux paramètres et conforté la cohérence du modèle standard. Il en existe néanmoins certains qui, dans la limite des incertitudes actuelles des mesures, contredisent des prédictions bien établies. Ainsi, la distribution angulaire des particules en fonction de la masse reconstruite de la paire de muons lors de désintégrations du type $\mathrm{B} \rightarrow \mathrm{K}^{\star} \mu^{+} \mu^{-}$est sensible à la présence de particules inconnues. De plus, les prédictions sur ces distributions sont relativement précises.

La comparaison entre les mesures et les estimations théoriques pour une de ces variables angulaires $\left(\mathrm{P}_{5}{ }_{5}\right)$ est visible sur la figure 6 et montre un désaccord. Il est possible que cette divergence soit due à une trop faible statistique ; seule l'accumulation des données par $\mathrm{LHCb}$ permettra de la confirmer ou de la réfuter. Mais il peut également s'agir d'une première preuve de la nature incomplète du modèle standard.

\section{Le futur de l'expérience}

L'accélérateur LHC doit redémarrer en 2015, avec un nombre de protons stockés dans la machine et une énergie accrus. Les expériences bénéficieront alors d'une plus grande statistique pour mesurer avec plus de précision les phénomènes qui se déroulent aux points de collisions protonproton.

Par ailleurs, les collaborations réfléchissent aux moyens d'améliorer la prise de données en augmentant l'efficacité des détecteurs et en changeant les méthodes de sélection des événements intéressants. Le prochain arrêt de l'accélérateur, prévu vers 2018, sera utilisé par la collaboration $\mathrm{LHCb}$ pour augmenter les performances de l'expérience, dans le but de gagner jusqu'à un ordre de grandeur sur la quantité de données utiles stockées.

Le modèle standard n'explique pas toutes les observations (l'exemple le plus flagrant est l'asymétrie matière-antimatière) ; la découverte d'une extension à ce modèle requiert plus de précision. C'est pourquoi un nombre croissant de physiciens travaillent en parallèle, à contraindre encore plus fortement le modèle standard en utilisant les données accumulées ces dernières années et, dans le même temps, à l'amélioration des détecteurs à venir. 